\title{
Hybrid Heuristics for the Linear Ordering Problem
}

\author{
Erik Garcia, Josu Ceberio ${ }^{\dagger}$ and Jose A. Lozano* ${ }^{\dagger}$ \\ *Basque Center for Applied Mathematics (BCAM), 48009 Bilbao, Spain \\ ${ }^{\dagger}$ Faculty of Computer Science, 20018 Donostia, Spain \\ University of the Basque Country UPV/EHU \\ email: erikg009@gmail.com, josu.ceberio@ehu.eus, ja.lozano@ehu.eus
}

\begin{abstract}
The linear ordering problem (LOP) is one of the classical NP-Hard combinatorial optimization problems. Motivated by the difficulty of solving it up to optimality, in recent decades a great number of heuristic and meta-heuristic algorithms have been proposed. Despite the continuous work on this problem, there is still room nowadays for designing strategies that beat the state-of-the-art algorithms, and take a step forward in terms of the quality of the obtained solutions.

In this paper, two novel schemes are presented. The first algorithm consists of an iterated local search algorithm that carries out an organized exploration of the search space. The second scheme is an extension of the previous algorithm that, based on the properties of the LOP, proposes an exact procedure that allows us to improve the quality of the solutions systematically. Conducted experiments on one of the hardest LOP benchmarks (xLOLIB) show that 77 new best results were found out of 78 instances. The described strategies also provide innovative ideas for developing more advanced algorithms for solving the LOP.
\end{abstract}

\section{INTRODUCTION}

Studied for the first time in 1958 by Chenery and Watanabe [1], the Linear Ordering Problem (LOP) [2], [3] has become one of the most classical combinatorial optimization problems. In 1979, Garey and Johnson [4] classified it as NP-hard and showeds the difficulty in solving LOP instances up to optimality. Despite being formalized a long time ago, it has received recent attention in the research community due to its numerous applications in diverse fields, such as archaeology [5], economics [6], graph theory [7], machine translation [8] or mathematical psychology [9].

As a result, the literature contains a wide variety of papers that have dealt with the LOP in its different representations and approaches. Most of the proposed algorithms can be classified in three different strategies: exact, heuristic and meta-heuristic. Among the exact methods, the most meaningful include Branch and Bound [10], [11], Branch and Cut [12] and Cutting Plane algorithms [13], [14]. When it comes to solving combinatorial optimization problems, apart from the exact methods that always solve the problem to optimality, there are heuristic and meta-heuristics that supply a good, although not always optimal, solution. Pioneering works proposed constructive heuristics [1], [15], [16]. Such approaches were later outperformed by the advances produced in meta-heuristic optimization. Proof of this is the solutions for the LOP based on Local Search [17], [18], Genetic Algorithms [19], Tabu Search [20], Scatter Search [21], Variable
Neighborhood Search [22], Ant Colony Optimisation [23], and recently Estimation of Distribution Algorithms [24].

According to a recent review of Marti et al. [25], the Memetic Algorithm (MA) and the Iterated Local Search (ILS) proposed by Schiavinotto et al. [26] and afterwards optimized by Ceberio et al. [3], are the algorithms that currently shape the state-of-the-art of the LOP. The MA is a hybrid algorithm which combines the canonical structure of a Genetic Algorithm with a high presence of local search procedures, either in the initialization of the population or in the evolutionary process itself. Alternatively, the ILS is a strategy that iteratively applies a local search algorithm to a single solution. When the process becomes trapped in a local optimal solution, the ILS applies a perturbation to the current solution, and continues with the optimization process until a termination criterion is satisfied. Both algorithms include an efficient implementation of a greedy local search algorithm.

Neither ILS nor MA explore the search space of solutions in an organized way. The exploration behavior of the ILS relies on the perturbation applied to the current solution. Similarly, the exploratory behavior of MA consists of the mutation of the solutions in the population by applying random modifications. Taking into account the factorial size of the search space of permutation problems, we think that the exploration behavior of ILS and MA can be further improved. Bearing that in mind, we propose an algorithm that explores the search space at different levels, starting from a more general exploration and delimiting slowly to local areas of the search space. This algorithm is called Hybrid Exploration Algorithm (HEA).

The second algorithm proposed in the paper is the Sequential Exact Improvement (SEI). This algorithm is based on the exact resolution of the LOP as a binary linear programming problem. When considering this type of approach, the exact solution can only be obtained for small instances $(n<75)$, and solving large instances (as in this work) is no longer feasible. Nevertheless, it is worth noting that the contribution of any item (or set of items) in the solution to the objective function is independent of the ordering of the previous and posterior items in the solution [3], [27]. This theoretical result permits the design of strategies that optimize the ordering of subsets of items (of size $n<75$ ) in the solution, and guarantees that the overall quality of the solution cannot worsen.

The conducted experiments show that the presented algo- 
rithms provide interesting procedures that allow us to outperform the best known results obtained for the LOP instances in XLOLIB.

The remainder of this paper is organized as follows. In Sect. II, we give a detailed description of the LOP, two different formalizations of the problem and some theoretical notes that will be used in Sect. III, where the two novel algorithms are presented. In Sect. IV, we carry out a thorough experimental analysis of the algorithms presented and compare the obtained results with the best known solutions. Finally, Sect. V sums up the main conclusions and raises some ideas for future work.

\section{The Linear Ordering Problem}

As pointed out in the introduction, the LOP was described for the first time in 1958, and throughout the following decades, a variety of representations have been published to formalize the problem. In what follows, we describe two possibilities that are used by the two algorithms proposed in the paper, respectively.

Given a square matrix $M=\left[m_{i, j}\right]_{i, j=1}^{n}$ of size $n$, the objective in the LOP is to find the joint permutation $\sigma=$ $\left(\sigma_{1}, \sigma_{2}, \ldots, \sigma_{n}\right)$ of rows and columns that maximizes the sum of the elements above the main diagonal. This objective can be formalized as maximizing the following objective function

$$
f(\sigma)=\sum_{i=1}^{n-1} \sum_{j=i+1}^{n} m_{\sigma_{i}, \sigma_{j}}
$$

The search space of solutions consists of all the permutations of the set of items $\{1,2, \ldots, n-1, n\}$, and the number of solutions is $n$ !.

A good point of the previous representation is that it is very intuitive, however, pioneering works tried to solve the problem by formulating it as a Binary Programming Problem [14]. This representation arises from the interpretation of the solutions of the LOP as directed acyclic complete graphs of $n$ nodes. In this interpretation, the graph is formed by putting a directed edge from node $i$ to node $j$ if the item $i$ is previous to the item $j$ in the solution. Any solution of the LOP can be expressed as a unique directed acyclic complete graph and any directed acyclic complete graph can be interpreted as a unique solution.

Once the solutions are understood as graphs, the proposal of [14] is the following. The graph is represented with the binary variables $x_{i j}$ of the problem which are the edges of the graph, 1 if there is an edge from node $i$ to node $j$ and 0 if there is no edge. So, the objective function to maximize is just $\sum_{i \neq j} x_{i j} m_{i j}$. The only remining aspects are the constraints needed to ensure that the binary variables form a directed acyclic complete graph, that is, a solution of the LOP.

The first constraint is to ensure the completeness of the graph, such that $x_{i j}+x_{j i}=1$ for each different pair of nodes. The second and third constraints are to ensure that there are no cycles, such that $x_{i j}+x_{j k}+x_{k i} \leq 2$ and $x_{i k}+x_{k j}+x_{j i} \leq 2$ for each different triple of nodes.

Summarizing, the LOP as a binary linear programming problem can be written as follows:

$$
\begin{aligned}
\max : \sum_{i \neq j} x_{i j} m_{i j} & \\
x_{i j}+x_{j i}=1, & \forall i, j \in V_{n}, i<j \\
x_{i j}+x_{j k}+x_{k i} \leq 2, & \forall i, j, k \in V_{n}, i<j, i<k, j<k \\
x_{i k}+x_{k j}+x_{j i} \leq 2, & \forall i, j, k \in V_{n}, i<j, i<k, j<k \\
x_{i j} \in\{0,1\}, & \forall i \neq j \in V_{n}
\end{aligned}
$$

where $V_{n}$ is the set of item/nodes $\{1,2, \ldots, n\}$.

\section{Hybrid HEURISTIC Algorithms}

In this section, the contribution of the paper, the two hybrid heuristic algorithms, are presented.

\section{A. Hybrid Exploration Algorithm}

As noted previously, in LOP, as in many other permutation problems, the search space of solutions is $n$ ! (all the permutations of size $n$ ), and thus, an exhaustive examination of all the solutions is not feasible beyond a small problem size $n$. As a consequence, most of the state-of-the-art designs try to find a balance between the exploration and exploitation abilities of the algorithms. In the case of the LOP, we think that strategies to balance that trade-off in the reference algorithms, ILS and MA [26], [3], can be further improved. On one hand, the exploration in ILS consists of a perturbation of the solution every time it reaches a local optimum. The idea is to escape from the local optimum and optimize other areas of the search space. On the other hand, MA is a population-based algorithm that executes a mutation operator that permits the addition of new random features to the solutions in the population. This procedure introduces diversity to the population and aims to explore new solutions.

Bearing this in mind, we present a simple meta-heuristic that faces the exploration of the search space by organizing the search at different levels: starting from a more broad exploratory behaviour and focusing it slowly to specific areas of the search space. This algorithm is called the Hybrid Exploration Algorithm (HEA). Note that even though in this work the aim is to optimize the LOP, the algorithm could be generalized to other problems where the solutions are represented as permutations or in general to other optimization problems with the correct adaptation.

According to the literature, using local search in the algorithm is almost mandatory to obtain high performances when solving the LOP. ILS is based on the local search and it explores the search space with perturbations that can be easily set to be larger or smaller. In addition, population-based algorithms, such as MA, are more likely to explore huge search spaces, therefore, we propose adapting the ILS to a populationbased algorithm and configuring the perturbations to perform the search in an organized manner.

The pseudocode of HEA is presented in Algorithm 1. The algorithm starts by building the initial population of Pop_size. To that end, it runs a stochastic variant of Becker's constructive heuristic (see section III-A1) as many times as there are solutions in the population. Afterwards, the local search algorithm under the insert neighborhood is applied to each of the solutions. 
Once the population has been initialized, the algorithm iterates on the next instructions. Firstly, the individuals of the population are moved (perturbed) with a certain number of swaps, and improved with a local search (under the insert neighborhood) to create an auxiliary population. Secondly, the best solutions of the newly created population and the former one are mixed, and the best Pop_size is chosen, while the rest of the solutions are discarded.

When a solution is perturbed considering a high number of swaps, it is expected that the obtained solution should be far from the one perturbed. Similarly, a low number of swaps results in a similar solution. Assuming this principle, we propose an algorithm that, at the beginning of the optimization, perturbs the solutions in the population with a high number of swaps in order to locate the regions with the best solutions in the search space, and, later, progressively decreases the number of swaps to explore in detail the chosen regions.

The number of random swaps starts with $0.45 n$ and decreases at a rate of $5 \%$ each time until $0.05 n^{1}$. In addition, for each number of swaps, the perturbation and improvement steps are repeated until there is no improvement in the highest value of the population $n \_s t o p$ consecutive times.

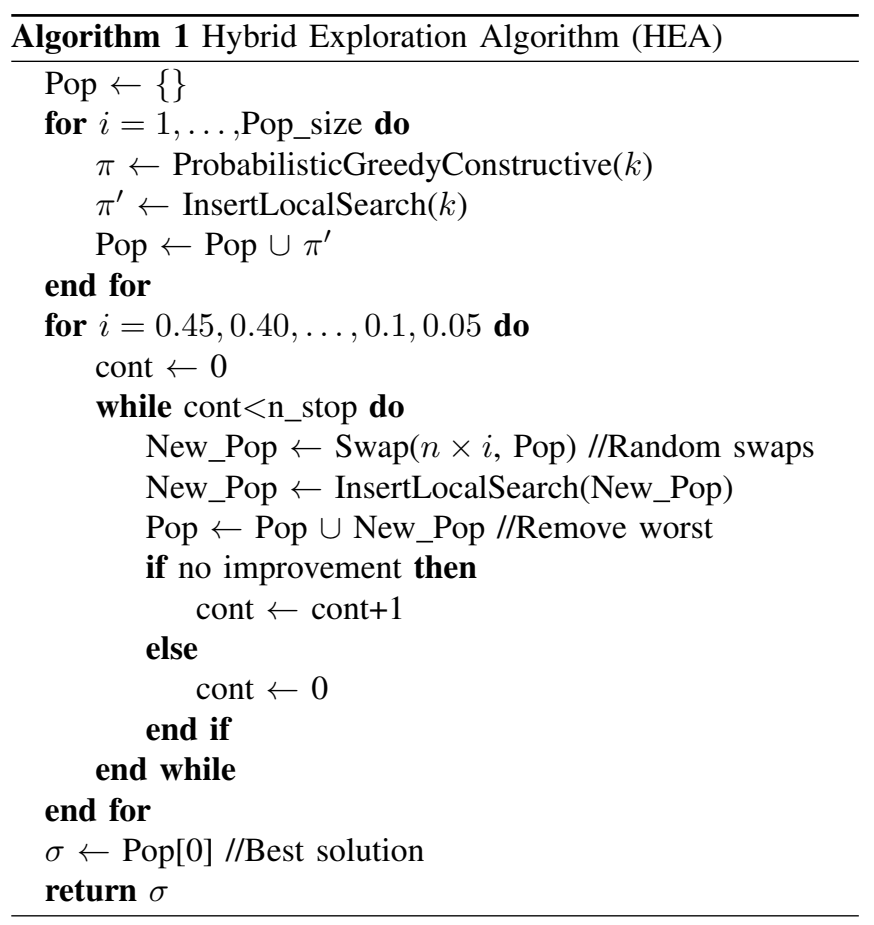

1) Probabilistic Greedy Constructive: The constructive heuristic used in HEA is a probabilistic version of the best known constructive algorithm for the LOP: the constructive of Becker [16]. This heuristic builds a solution by adding items one by one to it. At each time, the remaining items are ranked

\footnotetext{
${ }^{1}$ These parameters were deduced from a set of preliminary calibration experiments.
}

by a quotient

$$
q_{i}=\frac{\sum_{i \neq j} m_{i j}}{\sum_{i \neq j} m_{j i}}, \text { where } j \in\{\text { remaining items }\}
$$

which is the ratio between the contribution (sum of the parameters in the row) and non-contribution (sum of the parameters in the column) to the solution. As the contribution and non-contribution of the remaining items depend only on the remaining items, those items already added to the solution are not taken into account. At every iteration of the algorithm, the item with the largest quotient is added to the solution.

It is worth noting that the heuristic of Becker adds the item with the maximum quotient value to the construction at each time, so it always builds the same solution, i.e., the algorithm is deterministic.

The probabilistic version we propose consists of adding to the solution one of the best $k$ remaining items with a probability based on their quotient value. If the sum of the best $k$ items is $d=\sum_{\text {best } k} q_{i}$, then the probability to add one of these best $k$ items is $p_{i}=q_{i} / d$. Every time that the sum

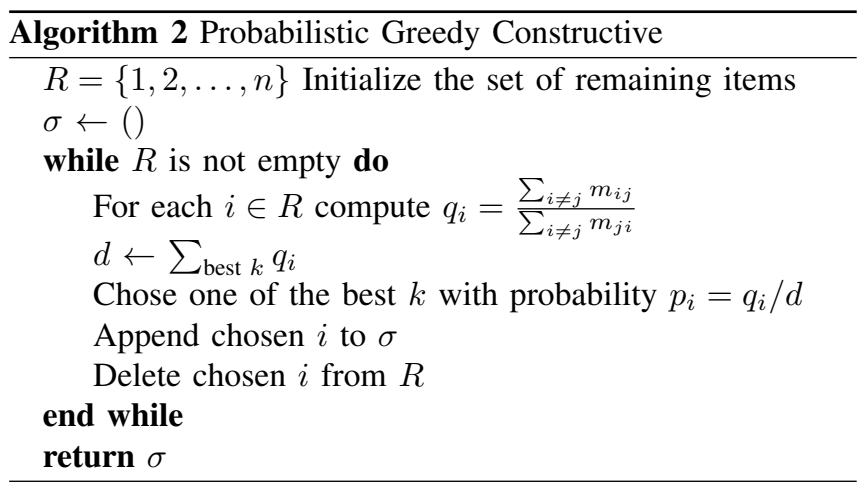

of parameters in the column of an item is zero, this is added to the solution automatically.

This procedure permits different solutions to be built every time the algorithm is run (with $k>1$ ). The pseudocode of the algorithm is described in Algorithm 2.

\section{B. Sequential Exact Improvement}

In general, when the Binary Linear Programming (BLP) representation is used to solve the LOP, the time needed to solve standard size problems is not affordable. Schiavinotto et al. [26] let an exact method run for one week for a matrix of 250 without getting any results. Although the exact solution cannot be obtained beyond certain values of $n$, due to the characteristics of the LOP, it is possible to use the BLP approach for improving the quality of solutions by solving submatrices of the problem. This is an innovative way of improving the solutions of the LOP that cannot be found in the literature, and can complement any algorithm already published for the LOP.

For a given solution $\sigma$, the contribution of any item at position $i$ is independent of the ordering of the items ordered in $\{1, \ldots, i-1\}$ positions, and also independent of the ordering of the items in $\{i+1, \ldots, n\}$ positions [3]. Not limited to 
that, the same idea can be extended to sets of items. So, the contribution of the items at positions from $i$ to $j$ is independent of the ordering of the items in $\{1, \ldots, i-1\}$ positions, and items in $\{j+1, \ldots, n\}$. As a result, if an optimization method is applied to the submatrix related to some contiguous items in the solution, based on the previous statements, it is guaranteed that the overall quality of the new solution can only improve, or remain equal if that subset of items is already optimal.

Let us consider, with illustrative purposes, the LOP instance in Fig. 1. Given the solution $\sigma=(2,3,1,4,5)$, if the contiguous numbers $(3,1,4)$ are considered, the exact solution of that submatrix inside the red square in Fig. 1 is $(3,4,1)$. In addition, we see that the contribution of item 2 (inside the

\begin{tabular}{|c|c|c|c|c|c|}
\hline & 2 & 3 & 1 & 4 & 5 \\
\hline 2 & 0 & 14 & 21 & 15 & 9 \\
\hline 3 & 23 & 0 & 26 & 26 & 12 \\
\hline & 16 & 11 & 0 & 15 & 7 \\
\hline & 22 & 11 & 22 & 0 & 13 \\
\hline & 28 & 25 & 30 & 24 & 0 \\
\hline
\end{tabular}

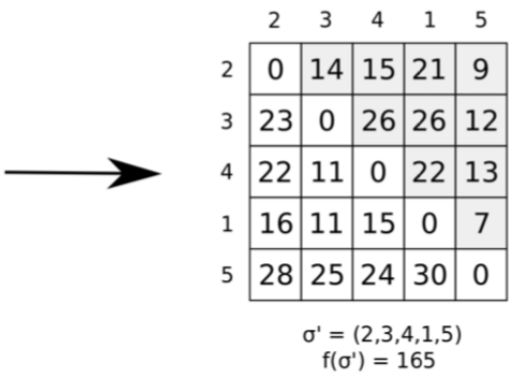

Fig. 1. Exact method applied to the contiguous subset of items $(3,1,4)$ to improve the current solution.

blue rectangle) before and after the optimization of the subset of items is the same. There is no contribution of item 5 as it is the last one, but the contributions of the posterior item, if they had existed, would have also remained the same. Only the contribution of the reordered items changes.

The previous remark on the LOP permits the improvement of any solution by applying optimization methods to submatrices of the problem. In this case, we propose a strategy to optimize contiguous submatrices in the solution following the BLP approach. In this sense, currently, there is also a huge variety of really competitive optimization solvers which have already implemented exact methods to solve these binary linear programs. Accordingly, taking that into account, in this work the commercial solver CPLEX has been used.

In order to provide intuition on the idea of solving contiguous matrices, we run some preliminary experiments with CPLEX on matrices of size 150 and 250 of the xLOLIB benchmark [26], and compared the results obtained with the best known results. From the conducted experiments, we conclude that:

- the algorithms based on solving continuous submatrices without any previous optimization are not competitive enough. So it is desirable to provide a good solution to the exact solver.

- Unless the size of the submatrix is large enough, the exact procedure does not contribute at all in the improvement of the solution.

- The time needed by the CPLEX to solve an instance of the LOP can be drastically reduced when the given

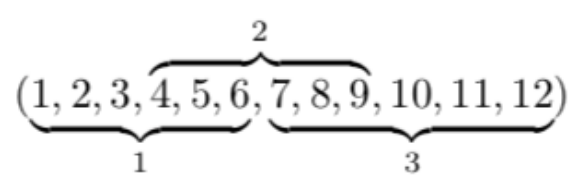

Fig. 2. Example of overlapping of submatrices of size 6 on the solution $(1,2,3,4,5,6,7,8,9,10,11,12)$

instance is a good solution.

In order to see the reduction of computational time required by CPLEX when a good solutions is provided, we conducted the following experiment. This experiment consists of solving matrices with the CPLEX, and once the solution is obtained, the matrices are reordered with the optimum solution obtained, and solved again with the CPLEX. Next, the execution times for solving the initial matrix and the reordered matrix are compared. Here we are reordering the matrices with the optimum solution (the CPLEX does not need to make any changes), but it can be considered quite similar to reordering them with a good solution that is not the optimum one, even if some small changes are needed. We used 10 matrices of size 50, 60 and 70, and this was built taking random rows and columns from some random instances of size 150 of xLOLIB. The results are provided in Table I.

TABLE I

THE TIMES TO SOLVE EACH OF THE 10 MATRICES OF SIZES 50, 60 AND 70 WITH CPLEX. THE FIRST LINE OF EACH SIZE IS THE TIME TAKEN TO SOLVE THE INITIAL MATRIX AND THE SECOND LINE IS THE TIME TAKEN TO SOLVE THE MATRIX REORDERED WITH THE OPTIMUM SOLUTION.

\begin{tabular}{|c|rrrrrrrrrr|}
\hline$n$ & \multicolumn{10}{|c|}{ Time(s) } \\
\hline 50 & 3 & 1 & 26 & 28 & 36 & 40 & 74 & 1 & 1 & 2 \\
& 1 & 1 & 10 & 19 & 11 & 23 & 27 & 1 & 1 & 2 \\
\hline 60 & 499 & 509 & 308 & 45 & 5 & 7 & 124 & 6 & 3 & 4 \\
& 221 & 68 & 121 & 26 & 4 & 4 & 31 & 4 & 2 & 3 \\
\hline 70 & 646 & 1855 & 3998 & 300 & 984 & 6185 & 1146 & 996 & 182 & 1378 \\
& 854 & 677 & 2437 & 599 & 163 & 5245 & 551 & 306 & 107 & 1047 \\
\hline
\end{tabular}

In the view of the results, we can conclude that when reordering the matrix with a good solution, the reduction of the time needed to solve it is significant (in the great majority of the instances).

Therefore, based on what has been exposed in previous paragraphs, we propose the Sequential Exact Improvement algorithm (SEI). This procedure is implemented to be used as a post-optimizer of another algorithm, i.e., HEA. For the sake of maximizing the probabilities of improving the solution given by HEA, we propose a sequential resolution of overlapped submatrices. For example, given the solution $(1,2,3,4,5,6,7,8,9,10,11,12)$ of Fig. 2, the algorithm would optimize first the submatrix from position 1 to 6 , secondly, the items from positions 4 to 9 , and finally from positions 7 to 12 . The idea of sequential overlapping submatrices permits any item to be moved to its optimal position. The process is repeated until no improvement is obtained. The pseudocode of SEI is presented in Algorithm 3. 


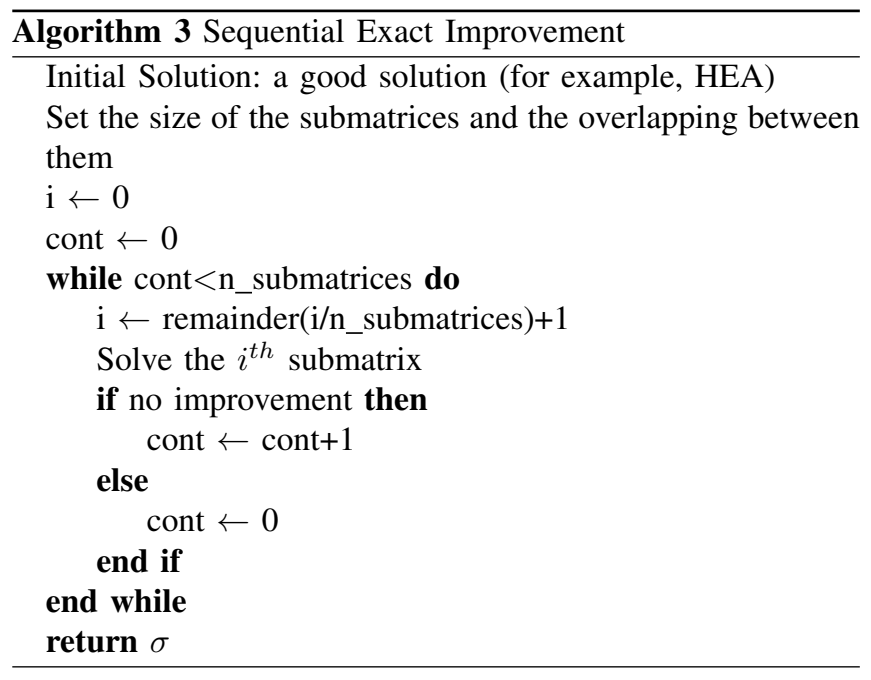

\section{EXPERIMENTAL STUDY}

With the aim of evaluating the validity of the proposed strategies, in this section we present a broad range of experiments. Particularly, the proposed algorithms are run on the xLOLIB benchmark of instances and the obtained results are compared with the best known results reported in the literature. This set of instances is composed of 39 instances of size 150 and 39 instances of size 250, and were generated by Schiavinotto et al. [26] through sampling uniformly at random the elements of the LOLIB instances. Currently, this benchmark constitutes the most challenging benchmark published on the LOP.

\section{A. Parameter Tuning}

The first step consists of setting up the two algorithms, that is, tuning their parameters.

1) Hybrid Exploration Algorithm: As regards HEA, there are three parameters to set: the number of items $k$ to consider in the probabilistic greedy constructive, the size of the population (Pop_size) and the number of consecutive nonimprovements iterations with the same best value of the population (n_stop).

In order to gain insights into the $k$ parameter, for each instance of xLOLIB we constructed 1000 solutions, with $k$ ranging from 1 to 20 . Obtained fitness values were averaged and normalized for each $k$ value. The results are presented in Fig. 3.

Bearing in mind Fig. 3, the best setting for the parameter is $k=1$, as the best normalized average values are obtained for most of the instances (close to 1). However, as explained above, with $k=1$ the algorithm becomes deterministic, and thus, since different solutions are needed to create an heterogeneous population, we set $k=5$, as it provides values close to 1 , but in the meantime, it has enough variance to build heterogeneous solutions.

In order to tune the population size and the stopping criterion, the other two parameters, 10 instances of the xLOLIB were randomly chosen, 5 instances of size 150 and another

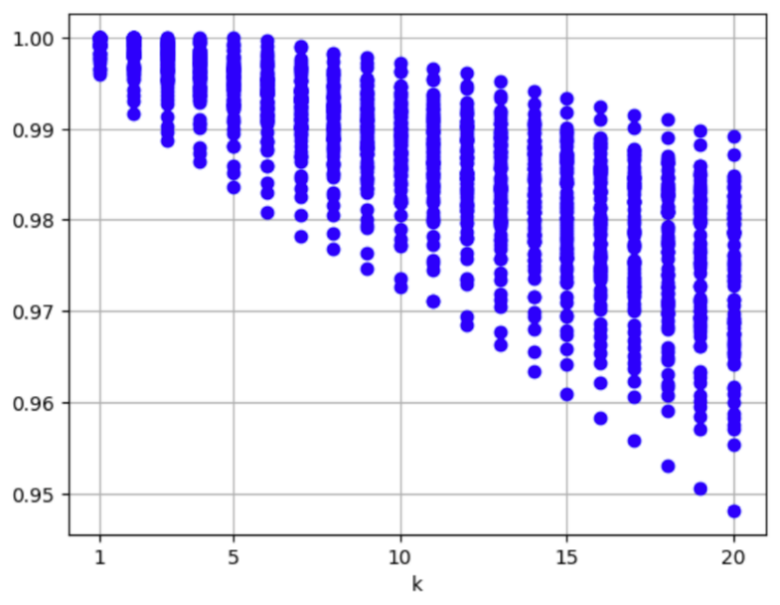

Fig. 3. The normalized fitness average values on the 78 instances of the xLOLIB for each value of $k$ from 1 to 20

5 of size 250 . In addition, $\{15,20,25,30\}$ population sizes and $\{25,50\}$ values for non-consecutive improvements were considered. 10 repetitions of HEA were performed for each combination of both parameters. The results are summarized in Table II in terms of average results of the obtained fitness values and execution time of 10 repetitions.

Looking at the results in Table II, the stopping criteria of 25 non-consecutive improvements is in most of the instances the best option (in 8 instances out of 10). In relation to the size of the population, it is not so clear, however, it looks like larger sizes obtained better results (in 6 instances out of 10 , the best population size is 30 ).

2) Sequential Exact Improvement: With respect to the setup of the sequential exact improvement algorithm, the structure of the sequence needs to be set, that is, the size of the submatrices to solve by CPLEX. As mentioned in previous experiments, the size of the submatrices has to be big enough in order to make an improvement. It is worth noting that for submatrices of size 50-60, they are actually optimal. Unfortunately, the computation time grows exponentially with the size of the submatrices (see Table I). According to the previous experiments, solving submatrices of size 80 is affordable in the majority of the cases, but not systematically in a reasonable time span (the computational time needed is excessive). So, the size of the submatrices is set to 75 as it supposes affordable execution times.

Once the size of the submatrices is set, an overlapping structure for the submatrices in instances of size 150 and 250 must be provided. We propose using the structures shown in Fig. 4 for each instance size. Given the $i^{\text {th }}$ submatrix, it overlaps the first half of items with the $(i-1)^{t h}$ submatrix, and similarly, the second half of items is overlapped with items that share with the $(i+1)^{t h}$ submatrix.

\section{B. Performance Evaluation}

Once the two algorithms has been set up, in the second step of the experimentation, the performance of both is measured 
TABLE II

AVERAGE FITNESS VALUE AND AVERAGE EXECUTION TIME OF 10 RUNS FOR EACH INSTANCE WITH DIFFERENT POSSIBLE COMBINATIONS OF 15, 20, 25, 30 POPULATION VALUES AND 25, 50 STOPPING CRITERIA VALUES. IN BOLDFACE WE HIGHLIGHT THE RESULTS THAT HAVE THE BEST TRADE-OFF OF AVERAGES.

\begin{tabular}{|c|c|c|c|c|c|c|c|c|c|}
\hline pop_size & Best Known & \multicolumn{2}{|c|}{15} & \multicolumn{2}{|c|}{20} & \multicolumn{2}{|c|}{25} & \multicolumn{2}{|c|}{30} \\
\hline n_stops & & 25 & 50 & 25 & 50 & 25 & 50 & 25 & 50 \\
\hline \multirow[t]{2}{*}{ N-be75np_150 } & 7172840 & 4508938 & 4508094 & 4508895 & 4510121 & 4509697 & 4509899 & 4509849 & 4510021 \\
\hline & & $38 \mathrm{~s}$ & $80 \mathrm{~s}$ & $49 s$ & $114 \mathrm{~s}$ & $62 s$ & $137 \mathrm{~s}$ & $74 s$ & $159 \mathrm{~s}$ \\
\hline \multirow[t]{2}{*}{ N-stabu1_150 } & 2874738 & 318945 & 318944 & 318934 & 318945 & 318947 & 318948 & 318935 & 318948 \\
\hline & & $34 s$ & $67 \mathrm{~s}$ & $44 s$ & $89 s$ & $58 \mathrm{~s}$ & $109 \mathrm{~s}$ & $69 s$ & $143 \mathrm{~s}$ \\
\hline \multirow[t]{2}{*}{ N-be75eec_150 } & 3480392 & 3157765 & 3155493 & 3157786 & 3158044 & 3157531 & 3157730 & 3158948 & 3158215 \\
\hline & & $42 s$ & $71 \mathrm{~s}$ & $54 \mathrm{~s}$ & $96 \mathrm{~s}$ & $60 \mathrm{~s}$ & $127 \mathrm{~s}$ & 81s & $146 \mathrm{~s}$ \\
\hline \multirow[t]{2}{*}{ N-be75tot_150 } & 12287935 & 1625807 & 1626068 & 1625665 & 1626036 & 1626035 & 1625995 & 1626286 & 1626087 \\
\hline & & $39 \mathrm{~s}$ & $77 \mathrm{~s}$ & $51 \mathrm{~s}$ & $92 \mathrm{~s}$ & $63 \mathrm{~s}$ & $131 \mathrm{~s}$ & $80 s$ & $144 \mathrm{~s}$ \\
\hline \multirow[t]{2}{*}{ N-be75oi_150 } & 2246282 & 957787 & 957903 & 957797 & 957887 & 957846 & 957832 & 957806 & 957884 \\
\hline & & $37 \mathrm{~s}$ & $80 s$ & $50 \mathrm{~s}$ & $107 \mathrm{~s}$ & $66 s$ & $127 \mathrm{~s}$ & $76 \mathrm{~s}$ & $141 \mathrm{~s}$ \\
\hline \multirow[t]{2}{*}{ N-be75oi_250 } & 5907714 & 17806671 & 17811018 & 17804801 & 17807416 & 17810906 & 17814331 & 17814316 & 17815642 \\
\hline & & $314 \mathrm{~s}$ & $644 \mathrm{~s}$ & $432 \mathrm{~s}$ & $834 \mathrm{~s}$ & $553 \mathrm{~s}$ & $1095 \mathrm{~s}$ & $659 \mathrm{~s}$ & $1281 \mathrm{~s}$ \\
\hline \multirow[t]{2}{*}{ N-be75eec_250 } & 8881423 & 5908086 & 5908937 & 5910149 & 5910700 & 5909920 & 5910521 & 5909914 & 5910219 \\
\hline & & $316 s$ & $672 \mathrm{~s}$ & $471 \mathrm{~s}$ & $853 \mathrm{~s}$ & $525 \mathrm{~s}$ & $1048 \mathrm{~s}$ & $636 \mathrm{~s}$ & $1265 \mathrm{~s}$ \\
\hline \multirow[t]{2}{*}{ N-be75np_250 } & 17796212 & 11903284 & 11904173 & 11904034 & 11905017 & 11904535 & 11904615 & 11904850 & 11903899 \\
\hline & & $278 \mathrm{~s}$ & $570 \mathrm{~s}$ & $415 \mathrm{~s}$ & $781 \mathrm{~s}$ & $501 \mathrm{~s}$ & $1026 \mathrm{~s}$ & $644 s$ & $1167 \mathrm{~s}$ \\
\hline \multirow[t]{2}{*}{ N-be75tot_250 } & 30934111 & 25405846 & 25411106 & 25400288 & 25404980 & 25407159 & 25412144 & 25410188 & 25411427 \\
\hline & & $316 \mathrm{~s}$ & $639 \mathrm{~s}$ & $410 \mathrm{~s}$ & $862 \mathrm{~s}$ & $526 \mathrm{~s}$ & $1087 \mathrm{~s}$ & $704 \mathrm{~s}$ & $1283 \mathrm{~s}$ \\
\hline \multirow[t]{2}{*}{ N-stabu1_250 } & 7734436 & 3057834 & 3057769 & 3058613 & 3059100 & 3058980 & 3058908 & 3059203 & 3059695 \\
\hline & & $308 \mathrm{~s}$ & $531 \mathrm{~s}$ & $424 \mathrm{~s}$ & $831 \mathrm{~s}$ & $521 \mathrm{~s}$ & $1046 \mathrm{~s}$ & 659 s & $1176 \mathrm{~s}$ \\
\hline
\end{tabular}

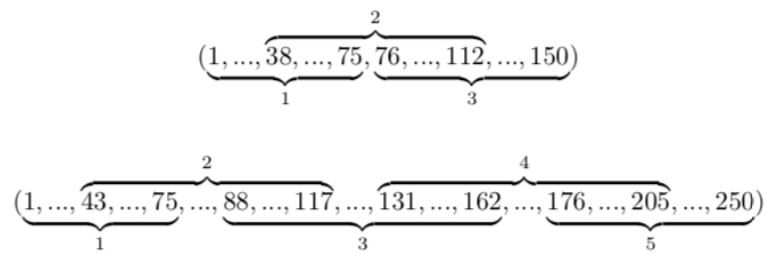

Fig. 4. Overlapping schemes of submatrices in instances of size 150 and 250.

and compared with respect to the best known results reported in the literature. Currently, the best known results for each instance of the XLOLIB are the maximal results obtained by Ceberio et al. [3] from 20 executions of the ILS $_{r}$ and $\mathrm{MA}_{r}$ using a stopping criteria of $10000 n^{2}$ evaluations.

We run the two algorithms, HEA and SEI, 10 repetitions on each instance from xLOLIB, and recorded the best and average fitness values of the obtained results. The results are presented in Table III. It is worth noting that, because of the nature of both algorithms and their stopping criterion, it makes not sense to stop the algorithm after a fixed number of function evaluations.

As can be seen, there are only a few best known solutions that are not outperformed by both algorithms. In fact, from 78 instances there is only one instance in which the best result is the one reported in the literature. Moreover, in 72 instances both algorithms obtained better best results than the state-of-the-art. Furthermore, another remarkable fact is that in 53 instances the obtained average value of both algorithms is better than the best known.

\section{CONClusions \& Future Work}

In this paper, two hybrid heuristic algorithms were proposed for solving a classical combinatorial optimization problem: the linear ordering problem. The first algorithm consists of an iterated local search algorithm under the insert neighbourhood that carries out an organized exploration of the search space. The second scheme is an extension of the previous algorithm that, based on the properties of the LOP, carries out an exact and sequential procedure that permits the quality of the solutions to be improved systematically. Conducted experiments on one of the hardest LOP benchmarks (xLOLIB) revealed an outstanding performance when compared to the existing best known solutions reported in the literature. Moreover, the experimentation concluded that there is still room nowadays for designing strategies that beat the state-of-theart algorithms, and take a step forward in terms of quality of obtained solutions.

For future research, there are a number of lines that would be interesting to explore. In this sense, one of them is to carry out a fair comparison between the hybrid exploration algorithm, the ILS and the MA with the execution time of the hybrid exploration algorithm (it stops when it finishes, the execution time cannot be set). This experiment would determine whether the organized exploration of the search space is more efficient than ILS or MA. Even so, there is practically no difference between the results of ILS or MA with the stopping criteria of $1000 n^{2}$ and $10000 n^{2}$ evaluations, so this suggests that there is not going to be an improvement, even if the stopping criteria (or execution time) is increased.

Another interesting research line would be the reduction of the execution time of the CPLEX when the matrix is reordered with a good solution as this allows larger submatrices to be solved, and thus, better results to be obtained. Moreover, in this paper we carried out a sequential procedure for applying CPLEX, other procedures nevertheless, such as a hierarchical one, could also be developed. 
TABLE III

RESULTS OF HEA AND SEI ON XLOLIB BENCHMARK. VALUES IN BOLDFACE HIGHLIGHT THE BEST RESULTS THAT OUTPERFORMED THE BEST KNOWN REPORTED FOR THAT INSTANCE.

\begin{tabular}{|c|c|c|c|c|c|c|c|c|c|c|}
\hline \multirow[b]{3}{*}{ Instance } & \multicolumn{5}{|c|}{$n=150$} & \multicolumn{5}{|c|}{$n=250$} \\
\hline & \multirow[b]{2}{*}{ Best Known } & \multicolumn{2}{|c|}{ HEA } & \multicolumn{2}{|c|}{ SEI } & \multirow[b]{2}{*}{ Best Known } & \multicolumn{2}{|c|}{ HEA } & \multicolumn{2}{|c|}{ SEI } \\
\hline & & Best & Average & Best & Average & & Best & Average & Best & Average \\
\hline N-be75eec & 3480392 & 3480968 & 3479751 & 3482828 & 3481407 & 8881423 & $\mathbf{8 8 9 2 9 9 5}$ & 8891153 & 8896595 & 8891954 \\
\hline N-be75np & 7172840 & 7182660 & 7172569 & 7174000 & 7171680 & 17796212 & 17822266 & 17811829 & 17822571 & 17815625 \\
\hline N-be75oi & 2246282 & 2246465 & 2246002 & 2246853 & 2246782 & 5907714 & 5911731 & 5910181 & 5912445 & 5910183 \\
\hline N-be75tot & 12287935 & 12287935 & 12281721 & 12287707 & 12275247 & 30934111 & 30985366 & 30972938 & 30998382 & 30973390 \\
\hline N-stabu 1 & 2874738 & 2875732 & 2874011 & 2875732 & 2873920 & 7734436 & 7743135 & 7738402 & 7747599 & 7740798 \\
\hline N-stabu2 & 4326696 & 4327108 & 4326312 & 4327538 & 4326382 & 11496181 & 11505756 & 11499498 & 11516097 & 11505425 \\
\hline N-stabu3 & 4508566 & 4510435 & 4509880 & 4510445 & 4510398 & 11895591 & 11909920 & 11903383 & 11909724 & 11907205 \\
\hline N-t59b11xx & 3234445 & 3239045 & 3235360 & 3239550 & 3237133 & 8402430 & 8409505 & 8403831 & 8411760 & 8408517 \\
\hline N-t59d11xx & 1461924 & 1462418 & 1462131 & 1462418 & 1462004 & 3837704 & 3842547 & 3838587 & 3843003 & 3838247 \\
\hline N-t59f11xx & 1542532 & 1543733 & 1540892 & 1543733 & 1541859 & 3984558 & 3995865 & 3992428 & 3995868 & 3991806 \\
\hline N-t59n11xx & 318967 & 318951 & 318946 & 318951 & 318948 & 823598 & 824974 & 824596 & 825023 & 824523 \\
\hline N-t65b11xx & 6448366 & 6454792 & 6452102 & 6455180 & 6453499 & 17248262 & 17267344 & 17261143 & 17275227 & 17261948 \\
\hline N-t65d11xx & 3556350 & 3559347 & 3559129 & 3559347 & 3558405 & 9342499 & 9352734 & 9349893 & 9353593 & 9350913 \\
\hline N-t65f11xx & 3158431 & 3159326 & 3157831 & 3159326 & 3158124 & 8406064 & 8417742 & 8411076 & 8414863 & 8411313 \\
\hline N-t65111xx & 253245 & 253224 & 253142 & 253417 & 253168 & 665926 & 666820 & 666548 & 666915 & 666412 \\
\hline N-t65n11xx & 550411 & 550893 & 550720 & 550893 & 550729 & 1429041 & 1430863 & 1429693 & 1430816 & 1429579 \\
\hline N-t70b11xx & 9645823 & 9644816 & 9637934 & 9645830 & 9637001 & 25387082 & 25415047 & 25410179 & 25416607 & 25412311 \\
\hline N-t70d11xn & 5823471 & 5825947 & 5825338 & 5825947 & 5825375 & 15195896 & 15206075 & 15201365 & 15211056 & 15204745 \\
\hline N-t70d11xx & 6172834 & 6173935 & 6170208 & 6174178 & 6168231 & 16037941 & 16042913 & 16035002 & 16043251 & 16036264 \\
\hline N-t70f11xx & 5149944 & 5150097 & 5147372 & 5150097 & 5147518 & 13575965 & 13588034 & 13580097 & 13598786 & 13588571 \\
\hline N-t70111xx & 436807 & 436862 & 436832 & 436862 & 436836 & 1112228 & 1112058 & 1111447 & 1114166 & 1112006 \\
\hline N-t70n11xx & 948721 & 948913 & 948879 & 948913 & 948776 & 2443584 & 2445125 & 2442707 & 2444720 & 2443509 \\
\hline N-t74d11xx & 9381843 & 9396044 & 9392875 & 9396044 & 9393381 & 24399174 & 24432273 & 24422273 & 24445112 & 24430106 \\
\hline N-t75d11xx & 9637128 & 9642140 & 9638921 & 9642140 & 9639000 & 25013216 & 25045329 & 25033516 & 25048727 & 25032804 \\
\hline N-t75e11xx & 41529895 & 41570193 & 41568469 & 41570193 & 41570193 & 106636934 & 106912584 & 106823295 & 106912899 & 106807717 \\
\hline N-t75k11xx & 1541226 & 1541596 & 1540908 & 1541596 & 1541462 & 4092119 & 4094732 & 4092379 & 4094468 & 4092300 \\
\hline N-t75n11xx & 1742437 & 1743094 & 1742710 & 1743094 & 1743094 & 4522276 & 4529853 & 4526019 & 4529018 & 4526330 \\
\hline N-tiw56n54 & 837257 & 837945 & 837412 & 837945 & 837479 & 2097731 & 2099727 & 2099079 & 2099727 & 2098696 \\
\hline N-tiw56n58 & 1155078 & 1155392 & 1154351 & 1155392 & 1154526 & 2902539 & 2906727 & 2905200 & 2906751 & 2906046 \\
\hline N-tiw56n62 & 1626118 & 1626528 & 1626170 & 1626495 & 1626243 & 4139627 & 4145747 & 4143253 & 4144996 & 4143041 \\
\hline N-tiw56n66 & 2107453 & 2107619 & 2107134 & 2107619 & 2107487 & 5366742 & $\mathbf{5 3 7 0 9 5 5}$ & 5368831 & $\mathbf{5 3 7 1 1 5 7}$ & 5369152 \\
\hline N-tiw56n67 & 2372706 & 2372945 & 2372678 & 2372945 & 2372930 & 6319305 & 6326441 & 6323465 & 6326881 & 6325307 \\
\hline N-tiw56n72 & 4135204 & 4135689 & 4133551 & 4135289 & 4133068 & 11146034 & 11156640 & 11150552 & 11153587 & 11150412 \\
\hline N-tiw56r54 & 957753 & 957966 & 957801 & 958060 & 957854 & 2385800 & 2387734 & 2386997 & 2388072 & 2387360 \\
\hline N-tiw56r72 & 2821686 & 2823758 & 2821757 & 2823758 & 2822299 & 7445814 & 7452983 & 7448311 & 7457217 & 7450081 \\
\hline
\end{tabular}

\section{ACKNOWLEDGMENTS}

This work has been partially supported by the Research Groups 2013-2018 (IT-609-13), BERC 2018-2021, and ELKARTEK programs (Basque Government), the projects TIN2016-78365-R (Spanish Ministry of Economy, Industry and Competitiveness) and Severo Ochoa Program SEV-20170718 (Spanish Ministry of Economy, Industry and Competitiveness).

\section{REFERENCES}

[1] H. B. Chenery and T. Watanabe, "International comparisons of the structure of production," Econometrica, vol. 26, no. 4, pp. 487-521, October 1958 .

[2] R. Martí and G. Reinelt, The linear ordering problem: exact and heuristic methods in combinatorial optimization. Springer, 2011, vol. 175

[3] J. Ceberio, A. Mendiburu, and J. A. Lozano, "The Linear Ordering Problem Revisited," European Journal of Operational Research, vol 241, no. 3, pp. 686-696, 2014.

[4] M. R. Garey and D. S. Johnson, Computers and Intractability: A Guide to the Theory of NP-Completeness. New York, NY, USA: W. H. Freeman \& Co., 1979.
[5] F. Glover, T. Klastorin, and D. Klingman, Optimal weighted ancestry relationships, ser. Management science report series. Business Research Division, Graduate School of Business Administration, University of Colorado, 1972.

[6] W. Leontief, Input-Output Economics. Cambridge University Press, 2008.

[7] I. Charon and O. Hudry, "A survey on the linear ordering problem for weighted or unweighted tournaments," 4or, vol. 5, no. 1, pp. 5-60, Mar. 2007.

[8] R. Tromble and J. Eisner, "Learning linear ordering problems for better translation," in Proceedings of the 2009 Conference on Empirical Methods in Natural Language Processing: Volume 2 - Volume 2, ser. EMNLP '09, 2009, pp. 1007-1016.

[9] J. G. Kemeny, "Mathematics without numbers," Daedalus, vol. 88, pp. $577-591,1959$.

[10] R. Kaas, "A branch and bound algorithm for the acyclic subgraph problem," European Journal of Operational Research, vol. 8, no. 4, pp. $355-362,1981$.

[11] I. Charon and O. Hudry, "A branch-and-bound algorithm to solve the linear ordering problem for weighted tournaments," Discrete Applied Mathematics, vol. 154, no. 15, pp. 2097 - 2116, 2006.

[12] M. Grötschel, M. Jünger, and G. Reinelt, "A cutting plane algorithm for the linear ordering problem," Operations research, vol. 32, no. 6, pp. 1195-1220, 1984.

[13] J. Mitchell and B. Borchers, "Solving real-world linear ordering prob- 
lems using a primal-dual interior point cutting plane method," Annals of Operations Research, vol. 62, no. 1, pp. 253-276, 1996.

[14] - "Solving linear ordering problems with a combined interior point/simplex cutting plane algorithm," in High Performance Optimization, ser. Applied Optimization, H. Frenk, K. Roos, T. Terlaky, and S. Zhang, Eds. Springer US, 2000, vol. 33, pp. 349-366.

[15] H. Aujac, "La hiérarchie des industries dans un tableau des échanges interindustriels," Revue économique, vol. 11, no. 2, pp. 169-238, 1960.

[16] O. Becker, "Das helmstädtersche reihenfolgeproblem - die effizien verschiedener näherungsverfahren -," in Computers Uses in the Social Sciences, Berichteiner Working Conference, Vienna, 1967.

[17] B. W. Kernighan and S. Lin, "An Efficient Heuristic Procedure for Partitioning Graphs," The Bell system technical journal, vol. 49, no. 1, pp. 291-307, 1970.

[18] S. Chanas and P. Kobylanski, "A new heuristic algorithm solving the linear ordering problem," Computational Optimization and Applications, vol. 6, no. 2, pp. 191-205, 1996.

[19] I. Charon and O. Hudry, "Lamarckian genetic algorithms applied to the aggregation of preferences," Annals of Operations Research, vol. 80, no. 0, pp. 281-297, 1998.

[20] M. Laguna, R. Marti, and V. Campos, "Intensification and diversification with elite tabu search solutions for the linear ordering problem," Computers \& Operations Research, vol. 26, pp. 1217-1230, 1999.
[21] V. Campos, F. Glover, M. Laguna, and R. Martí, "An experimental evaluation of a scatter search for the linear ordering problem," Journal of Global Optimization, vol. 21, no. 4, pp. 397-414, 2001.

[22] C. G. Garcia, D. Pérez-Brito, V. Campos, and R. Martí, "Variable neighborhood search for the linear ordering problem," Computers \& Operations Research, vol. 33, no. 12, pp. 3549 - 3565, 2006.

[23] C. Chira, C. M. Pintea, G. C. Crisan, and D. Dumitrescu, "Solving the linear ordering problem using ant models," in Proceedings of the 11th Annual conference on Genetic and evolutionary computation, ser. GECCO '09. New York, NY, USA: ACM, 2009, pp. 1803-1804.

[24] J. Ceberio, A. Mendiburu, and J. A. Lozano, "The plackett-luce ranking model on permutation-based optimization problems," in Evolutionary Computation (CEC), 2013 IEEE Congress on. IEEE, 2013, pp. 494501.

[25] R. Martí, G. Reinelt, and A. Duarte, "A benchmark library and a comparison of heuristic methods for the linear ordering problem," Comput. Optim. Appl., vol. 51, no. 3, pp. 1297-1317, Apr. 2012.

[26] T. Schiavinotto and T. Stützle, "The linear ordering problem: Instances, search space analysis and algorithms," Journal of Mathematical Modelling and Algorithms, vol. 3, no. 4, pp. 367-402, 2005.

[27] J. Ceberio, A. Mendiburu, and J. A. Lozano, "A note on the boltzmann distribution and the linear ordering problem," in Conference of the Spanish Association for Artificial Intelligence, 2016. 